\title{
Relationship between neuropsychology, clinical traits, psychopathology and attitudes towards change in eating disorders
}

Beatriz Mata-Saenz ( $\sim$ beamatasaenz@gmail.com )

Hospital General de Ciudad Real https://orcid.org/0000-0002-2587-4930

Teresa Rodríguez-Cano

Hospital General Universitario de Ciudad Real

Victoria Eugenia Muñoz-Martínez

Hospital General Universitario de Ciudad Real

Luis Beato-Fernández

Hospital General Universitario de Ciudad Real

Research article

Keywords: Neuropsychology, eating disorders, attention, executive functions, inhibition

Posted Date: April 24th, 2020

DOI: https://doi.org/10.21203/rs.3.rs-21795/v1

License: (a) (i) This work is licensed under a Creative Commons Attribution 4.0 International License.

Read Full License 


\section{Abstract}

Background:

Recent models try to find in eating disorders (ED) a relationship between the neuropsychological dimensions, the psychopathological disturbances and the clinical manifestations. Carrying out an investigation on neuropsychological aspects that may be related with an ED's characteristic cognitive pattern could contribute to their possible influence towards therapeutic change.

The main objective of this paper is to analyse the possible relationship between psychopathological and clinical variables with the neuropsychological characteristics of patients diagnosed with an ED and their possible influence on the attitude towards change.

Methods:

An observational analytical transversal study was done. Patients were outpatients referred during a 6month period who signed informed consent. Seventy-four subjects were included. Clinical (Psychiatric Status Rating Scale modified) and neuropsychological tests (Letter Number Sequencing; Stroop's test; Symbol and Digit Modalities Test; Rey-Osterrieth complex figure test) were administrated and psychopathological self-report questionnaires (Body Shape Questionnaire; Eating Disorders Inventory; The Bulimic Investigatory Test, Edinburgh; Beck's depression inventory; State-Trait Anxiety Inventory; Dissociative Experiences Scale; Attitudes Towards Change in Eating Disorders Scale) were given (but 23 participants, 31.08\%, did not give them back).

Results:

Nineteen patients $(25,68 \%)$ were diagnosed with restrictive anorexia nervosa (AN), 19 (25,68\%) purgative AN, 14 bulimia nervosa (18,92\%), 9 binge eating disorder (12,16\%) and 13 ED not otherwise specified $(17,57 \%)$. There are not any significant differences between the diagnoses with regards to the score of neuropsychological tests.

BMI was related to the majority of the neuropsychological scores. "Precontemplation" was related to time of copy ( $B=3.56$; IC 95\% (0.82-6.29), $p<0,01)$ and time of memory $(B=-2.31 ;$ IC 95\% $(-4.58-(-0.05)) ; p<0.05)$. "Contemplation" was related to time of copy $(B=1.43$; IC $95 \%(0.42-2.45) ; p<0.01)$ and index of style $(B=$ -14.01; IC 95\% (-24.98-(-3.04)); $p<0.01)$. "Decision" was related to working memory (SpanLN), quantitative score of copy, index of order and index of style.

Conclusions:

Neuropsychological alterations could be summarized as the working memory is lowered and the interference is increased, which is associated with a more controlling and less flexible attitude, and scores in inhibitory care are lower. ROCFT shows a visoperceptive deficit in some patients. 


\section{Background}

Eating disorders (ED) are characterized by the presence of multiple risk factors and their variable clinical course (1). Up to half of patients do not maintain diagnostic stability (2) which makes a standardization of the therapeutic approach difficult. Recent models try to find in the ED the relationship between, among other components, the neuropsychological dimensions and the psychopathological disturbances and the clinical manifestations of these disorders. A transdiagnosis perspective, in relation with possible endophenotypes and subphenotypes of the disorders, could reduce this high variability (3).

The transdiagnosis approach considers that there are some nuclear characteristics in all patients who suffer from an ED and that they could benefit from a common therapeutic approach (4). These characteristics are state-dependent, may probably appear in individuals who do not present active symptoms and would be shared by individuals at risk or their healthy relatives (4).

The identification of these endophenotypes has acquired great interest in recent years. Endophenotypic features of bulimia nervosa (BN) have been identified as the reduction in the activity of the serotonin transporter and certain cognitive and neuropsychological dimensions such as: executive functioning, changes in cognitive set shifting, lack of inhibition, or avoidant learning (4-6). In anorexia nervosa (AN) the most significant endophenotypes which stand out are those related to weight dysregulation and body image disturbance (4-6).

Carrying out an investigation on neuropsychological aspects that may be related with an ED's characteristic cognitive pattern could contribute to the identification of specific characteristics of these disorders and their possible influence towards therapeutic change.

Among the neuropsychological variables that may take up a relevant role in patients with an ED would be the ability to direct attention to target stimuli with the presence of distractors, memory, cognitive inflexibility and weak central coherence $(7,8)$. Of the above, cognitive inflexibility and weak central coherence could contribute to the perpetuation of the disorder, hindering mobilization to change (9-13). Cognitive rehabilitation programs with set shifting exercises, cognitive flexibility, global focus and searching for alternative solutions, would have some utility in promoting change (14).

Few studies explain the relationship between neuropsychological variables and eating psychopathology. This could be closer to a transdiagnostic view so it could be very interesting for these patients' treatment and recovery.

\section{Methods}

The main objective of this paper is to analyse the possible relationship between psychopathological and clinical variables with the neuropsychological characteristics of patients diagnosed with an ED as well as their possible influence on the attitude towards change. 
An observational analytical transversal study was done. Patients were outpatients referred from the Eating Disorders Unit of General University Hospital of Ciudad Real, during a 6-month period. Inclusion criteria were: (1) diagnosis of anorexia nervosa (AN), bulimia nervosa (BN), binge eating disorder (BED) or eating disorder not otherwise specified (EDNOS) according to the Diagnostic and Statistical Manual of Mental Disorders, Fifth Edition (DSM-5); (2) female gender; (3) aged between 18 and 50 years; (4) their current clinical state allows psychometric evaluation. Comorbidity with depression and anxiety was possible. Patients were excluded if they: 1) spoke a native language different from Spanish; (2) did not give written informed consent; (3) male gender; (4) comorbidity with bipolar disorder, psychotic disorder or major depressive disorder, organic mental disorder, attention-deficit hyperactivity disorder, autism spectrum disorder, Tourette syndrome or chronic fatigue syndrome.

\section{Participants}

At first, 74 subjects were included. The average age was 28.97 years (standard deviation, SD, 9.30). Upon arrival, participants were instructed about the procedure and signed informed consent. Clinical and neuropsychological tests were administrated and psychopathological self-report questionnaires were given (but 23 participants, $31.08 \%$, did not give them back).

Although the sample was reduced to 51 subjects, relationships between neuropsychological and clinical features and body shape disturbances (measured by Gardner Body Image Scale) could be evaluated in the 74 patients initially included (15).

\section{Procedure}

Treatment was conducted on an outpatient basis and followed the protocols of the unit with pharmacotherapy added to psychotherapy if it was needed. Motivational and cognitive- behavioural treatment was conducted individually, in groups and/or with family members.

Daily, selected patients were informed about the study and its procedure and informed consent was signed. After this, self-report questionnaires were given. Data were dissociated, anonymised and stored in a password-protected Excel base. Local Clinical Research Ethics Committee approved the study.

\section{Instruments}

Status

The patient's clinical status was evaluated by the Psychiatric Status Rating Scale. It is based on Herzog's work (16), updated with DSM-5 criteria for the different disorders. Patients would be divided into several groups according to punctuation: a score of 5-6 points mean patient met criteria of the disorder; 3-4 points mean partial recovery, and 1-2 points mean total recovery.

Body Shape Questionnaire (BSQ) 
Validation was carried out in the Spanish population by means of a 34-item self-report questionnaire with a six-point Likert-style rating scale ( $1=$ never; $6=$ always) $(17)$. It measures body weight and shape concern in ED. Its internal consistency using Cronbach was good (0.97) as well as its concurrent validity. It differentiates clinical from nonclinical subjects and persons with predictably more or less weight concern. A score of 105 is the cut-off point in the Spanish population.

Eating Disorders Inventory (2nd edition) (EDI-2)

This is a self-report questionnaire with 91 items (11 scales called: "Drive for the thinness", "Bulimia", "Body dissatisfaction", "Ineffectiveness", "Perfectionism”, "Interpersonal distrust”, "Interoceptive awareness", "Maturity fear", "Ascetism", "Impulsiveness" and "Social insecurity"). The internal consistency (Cronbach Alpha) is higher than 0.80 in ED samples. Reliability coefficients (alpha) were from 0.83 to 0.93 in samples of patients from the original studies. It is validated in the Spanish population $(18,19)$.

The Bulimic Investigatory Test, Edinburgh (BITE)

This is a self-reported test with 33 items to evaluate bulimic symptoms. There are two scales: "Symptoms" (30 items) and "Severity" ( 3 items) $(20,21)$. The Alpha coefficient is 0.96 for "Symptoms" and 0.62 for "Severity". Retest-reliability is also high (0.68-0.86).

Beck's depression inventory (BDI)

Self-reported questionnaire with 21 items to evaluate existence and severity of depressive symptoms $(22,23)$. Internal consistency is higher than 0.85 .

State-Trait Anxiety Inventory (STAI).

This is divided in two independent scales that measure state and trait anxiety (24-26). Cronbach's Alpha is 0.90 for trait anxiety and 0.94 for state anxiety.

Dissociative Experiences Scale (DES).

This is a self-assessment questionnaire based on a visual analogue scale composed of 28 dissociative experiences and asks how often a subject experiences them (0-100\%). An average of 30 or more means that dissociative disorder is expected (27-30). There are 3 scales: "Derealization and depersonalization", "Absorption" and "Amnesia" (31). The internal reliability (Cronbach's Alpha) was 0.93 and retestreliability 0.87 .

Attitudes Towards Change in Eating Disorders Scale (ACTA).

Self-reported questionnaire with 59 items validated in the Spanish population (32). There are 6 scales: "Precontemplation”, “Contemplation”, “Decision”, "Action”, “Maintenance” y "Relapse”. The reliability for each scale was from 0.90 to 0.74 and retest-reliability $0.86-0.64$. 
Neuropsychological variables:

\section{Working memory.}

This was measured by the test "Letter Number Sequencing" (LNSIII) included in WAIS-IV ("Wechsler Adult Intelligence Scale") (33). Participants listen to a verbal sequence of letters and numbers which is gradually increased and must be memorized, ordered and repeated back to the tester. Its reliability and validity are high, but data are related to intelligence evaluation.

Inhibition:

"Stroop's effect" (34), is defined as the difference in the process of naming colours and reading words. It defines Stroop's test, which measures inhibitory attention. Retest-reliability is high in all studies (0.690.89) (35).

\section{Sustained attention:}

Symbol and Digit Modalities Test (SDMT) $(36,37)$ mainly evaluates attention (included in the paradigm of sustained attention), optical tracking, speed of mental processing and visuo-motor speed. The test is based on pairing meaningless geometrical paintings with numbers from 1 to 9 following a previous model.

\section{Executive functions:}

The Rey-Osterrieth complex figure test (ROCFT) $(38,39)$ consists of copying and then drawing from memory a complicated line drawing. Several indexes are obtained: quantitative and qualitative scores of copying and memory reproduction accuracy, style, order and central coherence (40-42).

\section{Data analysis}

Statistical Package for the Social Sciences 19.0 was used for analysing the data obtained.

Firstly, a descriptive analysis was carried out, and after that an inferential one. The statistical level accepted as significant was $5 \%(p<0.05)$. Qualitative variables were statistically analysed by means of the Chi-Square test for independence. Normality was analysed for quantitative variables (KolmogorovSmirnov Test with more than 50 data and Shapiro-Wilk with less than 50 data). T-Student and U MannWhitney were used in dichotomic independent variables, and ANOVA and Kruskal-Wallis with independent variables with more than two categories. Pearson's and Spearman's correlations were used with quantitative independent variables.

Multivariate analysis was made by Simple Linear Regression to evaluate models of quantitative dependent variables. Variables included in the models were those which were statistically significant in bivariant analysis in our sample and variables statistically significant in previous studies or were considered of interest for the current study for their transdiagnostic meaning (Body Mass Index (BMI), 
dichotomic status, age of onset of ED, antidepressants, benzodiazepines, total score of DES, anxiety, depression, “Perfectionism”). Later, models were reduced to improve their alignment.

\section{Results}

Clinical, psychopathological and neuropsychological characteristics

Nineteen patients (25,68\%) were diagnosed with restrictive AN, 19 (25,68\%) purgative AN, 14 BN $(18,92 \%)$, 9 BED (12,16\%) and 13 EDNOS (17,57\%). The main results are summarized in Table 1.

Table 1: Qualitative clinical variables and their relationship with each clinical subtype: 


\begin{tabular}{|c|c|c|c|c|c|c|}
\hline Variable & $\begin{array}{l}\text { Total } \\
\text { sample } \\
\mathrm{N}=74 \\
\text { Fr (\%) / } \\
(\sigma)\end{array}$ & $\begin{array}{l}\text { Restrictive } \\
\text { AN } \\
\mathrm{n}=19 \\
\text { Fr (\%) / } \\
(\sigma)\end{array}$ & $\begin{array}{l}\text { Purgative } \\
\text { AN } \\
\mathrm{n}=19 \\
\text { Fr (\%) } / \square \\
(\sigma)\end{array}$ & $\begin{array}{l}\text { BN } \\
\mathrm{n}=14 \\
\mathrm{Fr}(\%) / \square \\
(\sigma)\end{array}$ & $\begin{array}{l}\text { BED } \\
\mathrm{n}=9 \\
\operatorname{Fr}(\%) / \square \\
(\sigma)\end{array}$ & $\begin{array}{l}\text { EDNOS } \\
\mathrm{n}=13 \\
\operatorname{Fr}(\%) / \square \\
(\sigma)\end{array}$ \\
\hline \multicolumn{7}{|l|}{ Compensatory behaviours } \\
\hline $\begin{array}{l}\text { Food restriction } \\
\text { Exercise } \\
\text { Laxatives } \\
\text { Vomiting } \\
\text { Diuretics } \\
\text { Diet pills }\end{array}$ & $\begin{array}{l}68 \\
(91.90 \%) \\
58 \\
(78.40 \%) \\
27 \\
(36.50 \%) \\
50 \\
(67.60 \%) \\
25 \\
(33.80 \%) \\
18 \\
(24.30 \%)\end{array}$ & $\begin{array}{l}19(100 \%) \\
15 \\
(78.90 \%) \\
3(57.90 \%) \\
11 \\
(15.80 \%) \\
4(21.10 \%) \\
1(5.30 \%)\end{array}$ & $\begin{array}{l}19(100 \%) \\
16(84.20 \%) \\
11(90 \%) \\
17(89.50 \%) \\
* * * \\
10(52.60 \%) \\
7(36.80 \%)\end{array}$ & $\begin{array}{l}13 \\
(92.90 \%) \\
11 \\
(78.60 \%) \\
9(64.30 \%) \\
13 \\
(92.90 \%) \\
* * * \\
6(42.90 \%) \\
7(50 \%) * *\end{array}$ & $\begin{array}{l}7 \\
(77.80 \%) \\
* * \\
7 \\
(77.80 \%) \\
0(0 \% * *) \\
1(11.10 \%) \\
2 \\
(22.20 \%) \\
2 \\
(22.20 \%)\end{array}$ & $\begin{array}{l}10 \\
(76.90 \%) \\
* * \\
9(69.20 \%) \\
4(30.80 \%) \\
8(61.50 \%) \\
3(23.10 \%) \\
1(7.70 \%)\end{array}$ \\
\hline Binges & $\begin{array}{l}18 \\
(75.70 \%)\end{array}$ & $8(42.10 \%)$ & $16(84.20 \%)$ & $\begin{array}{l}14 \\
(100 \% * * *)\end{array}$ & $\begin{array}{l}9(100 \%) \\
* * *\end{array}$ & $9(69.20 \%)$ \\
\hline Body mass index & $\begin{array}{l}21.97 \\
(7.45)\end{array}$ & $\begin{array}{r}17.69 \\
(2.56)\end{array}$ & $18.32(3.18)$ & $25.95(4.98)$ & $\begin{array}{l}34.15 \\
(9.98) *\end{array}$ & $\begin{array}{l}20.23 \\
(3.44)\end{array}$ \\
\hline $\begin{array}{l}\text { Time of evolution of illness } \\
\text { (years) } \\
\text { Pharmacotherapy }\end{array}$ & $\begin{array}{l}11.14 \\
(9.32) \\
52.70 \%\end{array}$ & $\begin{array}{l}10.95 \\
(9.48) \\
47.40 \%\end{array}$ & $\begin{array}{l}13.00 \\
(10.60) \\
73.70 \%\end{array}$ & $\begin{array}{l}10.21 \\
(7.83) \\
42.90 \%\end{array}$ & $\begin{array}{l}21.44 ; \sigma \\
9.82 \\
55.60 \%\end{array}$ & $\begin{array}{l}9.46 ; \sigma \\
4.99 \\
38.50 \%\end{array}$ \\
\hline Age of onset & $\begin{array}{l}16.62 \\
(6.54)\end{array}$ & $\begin{array}{l}17.05 \\
(7.01)\end{array}$ & $15.79(2.82)$ & $18.14(7.00)$ & $\begin{array}{l}11.89 \\
(3.79)\end{array}$ & $\begin{array}{l}18.85 \\
(9.24)\end{array}$ \\
\hline \multicolumn{7}{|l|}{ Clinical status } \\
\hline $\begin{array}{l}\mathrm{NS} \\
\mathrm{R} \\
\mathrm{PR} \\
\mathrm{M} \\
\mathrm{DC} \\
\mathrm{SC}\end{array}$ & $\begin{array}{l}9 \\
(12.20 \%) \\
12 \\
(16.20 \%) \\
15 \\
(20.30 \%) \\
11 \\
(14.90 \%) \\
18 \\
(24.30 \%) \\
9 \\
(12.20 \%)\end{array}$ & $\begin{array}{l}2(10.50 \%) \\
2(10.50 \%) \\
3(15.80 \%) \\
1(5.30 \%) \\
6(31.60 \%) \\
5(26.30 \%)\end{array}$ & $\begin{array}{l}1(5.30 \%) \\
2(10.50 \%) \\
3(15.80 \%) \\
3(15.80 \%) \\
6(31.60 \%) \\
4(21.10 \%)\end{array}$ & $\begin{array}{l}2(14.30 \%) \\
2(14.30 \%) \\
4(28.60 \%) \\
4(28.60 \%) \\
2(14.30 \%) \\
0(0 \%)\end{array}$ & $\begin{array}{l}1(11.10 \%) \\
2(22.20 \%) \\
2(22.20 \%) \\
3(33.30 \%) \\
1(11.10 \%) \\
0(0 \%)\end{array}$ & $\begin{array}{l}3(23.10 \%) \\
4(30.80 \%) \\
3(23.10 \%) \\
0(0 \%) \\
3(23.10 \%) \\
0(0 \%)\end{array}$ \\
\hline
\end{tabular}

NS: normal status; $R$ : residual; PR: partial remission; M: marked; DC: defined criteria; SC: severe defined criteria; ${ }^{*} p<0.05$ ${ }^{* *} p<0.01{ }^{* * *} p<0.001$. Fr: frequency $\left(\chi \square 40.619, g l 4{ }^{* * *} p<0.001\right)$

Neuropsychological variables are presented in Table 2. There are not any significant differences between the diagnoses with regards to the score of neuropsychological tests. The time of copy (ROCFT) was 
statistically significant related to dichotomic status $(Z-2,253, p<0,05$, average range 32,11 in patients without criteria of disorder vs 42.61 in patients with enough criteria).

Table 2: Neuropsychological variables:

\begin{tabular}{|c|c|c|c|}
\hline \multicolumn{4}{|c|}{ Variables $\quad$ Stadistics } \\
\hline \\
\hline & Mean & Median & SD \\
\hline Total LNS & 18.18 & 18.00 & 2.79 \\
\hline SPAN LN & 4.80 & 5.00 & 0.94 \\
\hline P-corrected by age & 100.97 & 108.00 & 26.43 \\
\hline C-corrected by age & 75.49 & 74.50 & 11.26 \\
\hline PC-corrected by age & 46.28 & 46.50 & 8.65 \\
\hline Interference & 4.50 & 3.25 & 11.83 \\
\hline SDMT score & 54.64 & 55.00 & 11.36 \\
\hline $\begin{array}{l}\text { ROCFT copy time } \\
\text { (min) }\end{array}$ & 3.07 & 3.00 & 2.04 \\
\hline $\begin{array}{l}\text { ROCFT copy } \\
\text { quantitative (direct) }\end{array}$ & 34.45 & 35.00 & 2.46 \\
\hline $\begin{array}{l}\text { ROCFT memory time } \\
(\mathrm{min})\end{array}$ & 2.72 & 2.00 & 1.52 \\
\hline $\begin{array}{l}\text { ROCFT memory } \\
\text { quantitative (direct) }\end{array}$ & 19.60 & 20.00 & 6.14 \\
\hline Order index & 2.23 & 2.33 & 0.67 \\
\hline Style index & 1.56 & 1.67 & 0.29 \\
\hline $\begin{array}{l}\text { Central coherence } \\
\text { index }\end{array}$ & 1.45 & 1.52 & 0.32 \\
\hline \multicolumn{4}{|l|}{ Qualitative variables } \\
\hline Scaled score SDMT & \multicolumn{3}{|c|}{ Very low 8 (10.81\%); Low 2 (2.70\%); Normal 51 (68.92\%); High 13 (17.57\%) } \\
\hline ROCFT copy types & \multicolumn{3}{|c|}{ | 46 (62.16\%); || 10 (13.51\%); ||I 5 (6.76\%); IV 11 (14.86\%); V 1 (1.35\%); VI 1 (1.35\%) } \\
\hline ROCFT memory types & \multicolumn{3}{|c|}{ I 58 (78.38\%); || 7 (9.46\%); || 1 (1.35\%); IV 5 (6.76\%); V 1 (1.35\%); VI 2 (2.70\%) } \\
\hline ROCFT observations & \multicolumn{3}{|c|}{$\begin{array}{l}\text { No observations } 53 \text { (71.62\%); Completion } 6 \text { (8.11\%); Poorness } 5 \text { (6.76\%); Child/Perfectionism/Repeated } \\
\text { lines/Very small/Unconnected } 2(2.70 \%) \text { (each one) }\end{array}$} \\
\hline
\end{tabular}

In Table 3 the main psychopathological results are shown. When the patients who did not fill in the questionnaires $(23,31 \%, n=23)$ were compared to ones who did, no differences were found in age, $B M I$, age of onset, time of evolution, level of studies, use of drugs, diagnoses, compensatory symptoms or neuropsychological scores. 
Table 3: Variables of psychopathological tests:

\begin{tabular}{llll}
\hline Variable & Mean & Median & SD \\
\hline ACTA & & & \\
Precontemplation & 10.73 & 10.00 & 8.46 \\
Contemplation & 16.42 & 16.36 & 9.34 \\
Decission & 17.22 & 17.27 & 94.41 \\
Action & 26.04 & 26.00 & 7.64 \\
Maintenance & 14.86 & 15.00 & 9.00 \\
Relapse & 17.51 & 15.71 & 11.37 \\
EDI-2 & & & \\
DT & 11.61 & 12.00 & 6.77 \\
B & 3.61 & 3.00 & 5.23 \\
BD & 14.76 & 15.00 & 8.88 \\
I & 11.37 & 9.00 & 8.33 \\
P & 7.22 & 7.00 & 3.85 \\
ID & 5.86 & 5.00 & 4.44 \\
IA & 10.57 & 10.00 & 7.71 \\
MF & 8.78 & 6.00 & 6.22 \\
A & 6.49 & 5.00 & 5.00 \\
IR & 6.18 & 5.00 & 10.49 \\
SI & 8.65 & 9.00 & 5.69 \\
BITE & & & \\
BITE-síntomas & 15.31 & 14.00 & 8.51 \\
BITE-gravedad & 7.90 & 6.00 & 7.29 \\
BITE-suma & 23.22 & 21.00 & 14.43 \\
BSQ & 126.16 & 123.00 & 45.59 \\
DES & & & \\
DES-amnesia & 6.67 & 3.13 & 10.49 \\
DES-absorption & 22.66 & 21.11 & 21.63 \\
DES- depersonalization & 10.09 & 0.83 & 17.95 \\
DES-total & 14.16 & 8.93 & 15.17 \\
BDI & 20.88 & 22.00 & 10.34 \\
STAI & & & \\
STAl-state & 30.69 & 31.00 & 14.00 \\
STAl-trait & 35.92 & 35.00 & 13.26 \\
\hline & & & \\
\hline & & &
\end{tabular}

DT: Drive for the thinness; B: Bulimia; BD: Body dissatisfaction; I: Ineffectiveness; P: Perfectionism; ID: Interpersonal distrust; IA: Interoceptive awareness; MF: Maturity fear; A: Ascetism; IR: Impulsiveness; SI: Social insecurity.

Table 4 shows the main relationships between clinical and neuropsychological variables. BMI was related to the majority of the neuropsychological scores, followed by time of evolution and age of onset. 
Table 4: Statistically significant relationship between clinical and neuropsychological variables:

\begin{tabular}{|c|c|c|c|}
\hline Relationship & Variable & Test & Stadistics \\
\hline Total LNS & $\mathrm{BMI} * 1$ & $\begin{array}{l}\text { rho } \\
\text { Spearman }\end{array}$ & Coef 0.40 \\
\hline P-corrected by age & Age of onset* & $\begin{array}{l}\text { rho } \\
\text { Spearman }\end{array}$ & Coef 0.34 \\
\hline C-corrected by age & Age of onset* & $\begin{array}{l}\text { rho } \\
\text { Spearman }\end{array}$ & Coef 0.31 \\
\hline \multirow[t]{5}{*}{ SDMT score } & Age of onset* & $\begin{array}{l}\text { rho } \\
\text { Spearman }\end{array}$ & Coef 0.29 \\
\hline & Time of evolution of illness* & $\begin{array}{l}\text { rho } \\
\text { Spearman }\end{array}$ & Coef -0.33 \\
\hline & Dicotomic time of evolution of illness * & U-MW & $\begin{array}{l}Z-2.403 \\
\text { Average range } 43.34 \text { vs } \\
31.33\end{array}$ \\
\hline & Use of drugs** & t-Student & $\begin{array}{l}\text { F } 1.530 \\
\text { Average } 50.38 \text { vs } 59.37\end{array}$ \\
\hline & $\begin{array}{l}\text { BMI intervals*2 } \\
\mathrm{BMI}^{\star 1}\end{array}$ & $\begin{array}{l}\text { ANOVA } \\
\text { rho } \\
\text { Spearman }\end{array}$ & $\begin{array}{l}\mathrm{F} 3.02, \mathrm{gl} 3 \\
\text { Coef } 0.38\end{array}$ \\
\hline \multirow[t]{3}{*}{ Scaled SDMT } & $\begin{array}{l}\text { Dicotomic time of evolution of illness } \\
\star 1\end{array}$ & $\chi^{2}$ & $\chi^{2} 8.98, \mathrm{gl} 3$ \\
\hline & Use of drugs ${ }^{2}$ & $\chi^{2}$ & $\chi^{2} 10.68, \mathrm{gl} 3$ \\
\hline & $\begin{array}{l}\text { Dicotomic time of evolution of illness } \\
\star 2\end{array}$ & $\chi^{2}$ & $\chi^{2} 19.10$, gl 9 \\
\hline \multirow[t]{3}{*}{ ROCFT copy time (min) } & Use of drugs $\star \star \star ~$ & U-MW & $\begin{array}{l}Z-1.948 \\
\text { Average range } 32.71 \text { vs } \\
41.79\end{array}$ \\
\hline & Dicotomic status* & U-MW & $\begin{array}{l}Z-2.25 \\
\text { Average range } 32.11 \mathrm{vs} \\
42.61\end{array}$ \\
\hline & $\mathrm{BMI} \star 2$ & $\begin{array}{l}\text { rho } \\
\text { Spearman }\end{array}$ & Coef -0.28 \\
\hline ROCFT memory time (min) & $\mathrm{BMI} * 2$ & $\begin{array}{l}\text { rho } \\
\text { Spearman }\end{array}$ & Coef -0.31 \\
\hline \multirow{2}{*}{$\begin{array}{l}\text { ROCFT memory quantitative } \\
\text { (direct) }\end{array}$} & BMl intervals* & ANOVA & $\mathrm{F} 3.43, \mathrm{gl} 3$ \\
\hline & $\mathrm{BMI} * 1$ & $\begin{array}{l}\text { rho } \\
\text { Spearman }\end{array}$ & Coef 0.37 \\
\hline
\end{tabular}

U-MW: U-Mann Whitney, $\chi^{2}$ : chi-square; Coef: correlation coefficient; * $p<0.05 ;{ }^{*} \mathrm{p}<0.001 ;{ }^{* \star *} \mathrm{p}=0.051 ;{ }^{1}$ Only AN and BN; ${ }^{2}$ The relationship disappeared when the sample was reduced to $\mathrm{AN}$ and $\mathrm{BN}$

The statistically significant correlations between psychopathological and neuropsychological tests are summarized in Table 5. When the sample was reduced to AN and BN participants, no significant relationships between depression or anxiety and neuropsychological variables were found. 
Table 5: Statistically significant correlations between neuropsychological and psychopathological variables

\begin{tabular}{llll}
\hline Variable & Related Variable & Test & Stadistics \\
\hline Total LNS & Maturity fear & Spearman & rho -0.29 \\
SpanLN & Decission & Spearman & rho -0.29 \\
Interference & Perfectionism & Spearman & rho 0.03 \\
& DES-absorption & Spearman & rho 0.34 \\
& DES-total & Spearman & rho 0.32 \\
SDMT & Depression & U-Mann & no-depression average range 32.64 vs depression average \\
& Maintenance & Whitney & range 23.49 \\
ROCFT copy & Spearman & rho -0.33 \\
quantitative & Relapse & Spearman & rho 0.28 \\
& Interoceptive & Spearman & rho 0.29 \\
& awareness & & \\
& Social insecurity & Spearman & rho 0.30 \\
& STAl-trait & Spearman & rho 0.28 \\
ROCFT copy qualitative & Contemplative & ANOVA & F 2.63 gl 5 \\
& Action & ANOVA & F $3.23 \mathrm{gl} 5$ \\
& Drive for the & Kruskal- & Chi 11.81 \\
thinness & Wallis & \\
ROCFT memory & DES-amnesia & Spearman & rho 0.30 \\
quantitative & Action & Kruskal- & Chi 12.36 \\
ROCFT memory & Contemplative & Spearman & rho 0.28 \\
qualitative & Decission & Spearman & rho 0.31 \\
Order index & Spearman & rho 0.30 \\
& &
\end{tabular}

$p<0.05$

Relationship between neuropsychological variables and attitude to the change

"Precontemplation" was related to time of copy ( $B=3.56$; IC 95\% (0.82-6.29), $p<0,01)$ and time of memory $(B=-2.31$; IC 95\% (-4.58-(-0.05)); $p<0.05)$. “Contemplation” was related to time of copy $(B=1.43 ;$ IC $95 \%$ $(0.42-2.45) ; p<0.01)$ and index of style $(B=-14.01 ;$ IC 95\% (-24.98-(-3.04)); $p<0.01)$. "Decision" was related to working memory (SpanLN), quantitative score of copy, index of order and index of style.

\section{Multiple lineal regression analysis}


The last models are presented in Table 6 .

Table 6: Multiple lineal regression models: 


\begin{tabular}{|c|c|c|c|c|}
\hline Dimenssion & Dependent variable & Independent variable & $\mathrm{B}(\mathrm{IC})$ & $p$ \\
\hline \multirow[t]{17}{*}{ Working memory } & \multirow[t]{10}{*}{ Total LNS } & Maturity fear & $-0.02(-0.14-0.10)$ & 0.73 \\
\hline & & Depression & $-0.26(-0.38-(-0.14))$ & $<0.001$ \\
\hline & & STAI-state & $0.57(-0.02-0.14)$ & 0.16 \\
\hline & & STAI-trait & $0.07(-0.04-0.18)$ & 0.20 \\
\hline & & Perfectionism & $0.08(-0.10-0.25)$ & 0.39 \\
\hline & & Dicotomic status & $1.52(0.11-2.92)$ & 0.03 \\
\hline & & DES-total & $0.04(-0.01-0.09)$ & 0.09 \\
\hline & & Antidepressants & $0.65(-0.60-1.91)$ & 0.30 \\
\hline & & Age of onset & $0.07(-0.02-0.16)$ & 0.12 \\
\hline & & $\mathrm{BMI}$ & $0.13(0.04-0.21)$ & 0.004 \\
\hline & \multirow[t]{7}{*}{ SpanLN } & Depression & $-0.07(-0.11-(-0.03))$ & 0.001 \\
\hline & & STAI-trait & $0.02(-0.02-0.05)$ & 0.29 \\
\hline & & Perfectionism & $0.06(-0.00-0.13)$ & 0.05 \\
\hline & & Dicotomic status & $0.66(0.15-1.17)$ & 0.01 \\
\hline & & DES-total & $0.02(0.00-0.04)$ & 0.047 \\
\hline & & Age of onset & $0.03(-0.00-0.06)$ & 0.09 \\
\hline & & BMl & $0,02(-0,01-0,06)$ & 0.11 \\
\hline \multirow[t]{7}{*}{ Inhibitory attention } & \multirow[t]{7}{*}{ Interference } & Depression & $-0.26(-0.68-0.09)$ & 0.13 \\
\hline & & Perfectionism & $1.07(0.16-1.99)$ & 0.02 \\
\hline & & DES-total & $-0.45(-1.06-0.15)$ & 0.14 \\
\hline & & Antidepressants & $1.42(-4.99-7.82)$ & 0.66 \\
\hline & & BMI & $-0.43(-0.89-0.03)$ & 0.07 \\
\hline & & DES-absorption & $0.41(0.01-0.82)$ & 0.04 \\
\hline & & Binges & $7.16(-0.59-14.91)$ & 0.07 \\
\hline \multirow[t]{8}{*}{ Sustained attention } & \multirow[t]{8}{*}{ SDMT score } & Depression & $-0.95(-1.49-(0.40))$ & 0.001 \\
\hline & & STAI-trait & $0.46(0.42-0.88)$ & 0.03 \\
\hline & & Perfectionism & $1.09(0.31-1.87)$ & 0.01 \\
\hline & & DES-total & $0.15(-0.06-0.36)$ & 0.16 \\
\hline & & Antidepressants & $-1.49(-7.59-4.61)$ & 0.62 \\
\hline & & Sedative-hypnotic & $-6.24(-13.08-0.60)$ & 0.07 \\
\hline & & Age of onset & $0,30(-0,09-0,69)$ & 0.13 \\
\hline & & BMl & $-0.12(-0.49-0.26)$ & 0.53 \\
\hline \multirow[t]{17}{*}{ Executive functions } & \multirow[t]{6}{*}{ ROCFT time of copy } & STAI-state & $-0.04(-0.08-0.00)$ & 0.05 \\
\hline & & STAI-trait & $0.02(-0.02-0.07)$ & 0.26 \\
\hline & & Perfectionism & $0.03(-0.06-0.11)$ & 0.52 \\
\hline & & Dicotomic status & $0.41(-0.23-1.05)$ & 0.21 \\
\hline & & Antidepressants & $0.49(-0.12-1.11)$ & 0.11 \\
\hline & & BMl & $-0.04(-0.08-0.00)$ & 0.06 \\
\hline & \multirow[t]{6}{*}{ ROCFT copy quantitative } & Depression & $-0.13(-0.27-0.01)$ & 0.06 \\
\hline & & STAI-trait & $0.10(-0.00-0.20)$ & 0.06 \\
\hline & & Perfectionism & $0.20(0.01-0.40)$ & 0.04 \\
\hline & & Sedative-hypnotic & $-1.10(-2.71-0.51)$ & 0.18 \\
\hline & & Age of onset & $-0.11(-0.21-(-0.01))$ & 0.03 \\
\hline & & BMl & $-0.03(-0.12-0.07)$ & 0.57 \\
\hline & \multirow[t]{5}{*}{ Central coherence index } & Perfectionism & $0.02(-0.01-0.04)$ & 0.17 \\
\hline & & Dicotomic status & $-0.11(-0.30-0.08)$ & 0.27 \\
\hline & & Antidepressants & $0.25(0.05-0.46)$ & 0.02 \\
\hline & & Sedative-hypnotic & $-0.25(-0.48-(-0.02))$ & 0.03 \\
\hline & & Antipsychotics & $0.59(0.08-1.10)$ & 0.02 \\
\hline
\end{tabular}


B: beta coefficient; IC: 95\% confidence interval; p: level of statistical significance

\section{Discussion}

The main findings of the present work show that ED clinical subtypes do not significantly differ regarding to neuropsychological features. Overall, there seems to be a relationship between some results in neuropsychological tests and certain clinical characteristics, mainly the BMI, time of evolution of the disorder, age of onset and clinical status. Pharmacological treatment, another influence, could be considered as a confounding factor. Regarding psychopathological features, a trait with endophenotypic value, perfectionism, is associated with the capacity for sustained attention, interference and some executive functions, such as planning capacity and perfectionism evaluated with ROCFT copy scores.

In turn, executive functions can influence attitude towards change, especially in terms of planning and abstraction of medium-long term consequences or from analysis and capacity and insight.

These results are relevant because of the possible influence over functional recovery.

Neuropsychological changes in ED and related clinical and psychological factors.

Working memory.

Working memory was reduced in patients of this study, in relation to the general population (an average SpanLN between 5.7-4.9 with a $\sigma$ of 1.0 would be expected and an average of 4 was found, 8 with a $\sigma$ of 0.94). Other authors (43) who exclusively explored patients with an AN diagnosis, found even lower scores, which could be explained by the existence of some degree of worsening (43-45).

Clinical status, BMI, depression and dissociation, total scores, are the factors that are most associated with working memory scores. Use of pharmacological treatments would be a confusing variable as is shown in Table 6.

Data reported in literature related to working memory in ED are contradictory (43). Until now, we do not know of any study that simultaneously considers all the analysed factors in the model of the present work. Previous work shows the necessity of regarding factors like comorbid pathology, time of evolution or the clinical status of patients (46) which are included in our model.

Attention.

The inhibitory attention, assessed by the Stroop interference, was higher than the expected score (mean $4.50, \sigma 11.83$ versus an expected score of $2.71, \sigma 9.05)(35)$. Inhibitory attention has been related to neuroticism and a more controlling and less flexible attitude (43). 
Sustained attention was preserved or high in $86 \%$ of the patients. Factors most related to this variable in the present work are perfectionism, anxiety and depression. It is worth noting the absence of a relationship between eating pathology and sustained attention, unlike the mentioned clinical variables.

Other authors have also related attentional disturbances with clinical variables (clinical status, BMI, time of evolution, drugs) rather than eating disorders $(43,47,48)$. Furthermore, attention deficits have been related to deficits in visual processing (43). The interest of the present work is that a variable not studied in the mentioned works and with a transdiagnostic value, such as perfectionism, is associated with sustained attention, in addition to anxiety and depression.

Executive Functions.

Perfectionism, cognitive inflexibility, the need for control in matters related to food and the difficulty in developing alternatives to manage everyday problems are frequent characteristics of people with eating disorders (49). All this has led to the proposal that there may be deficits in the paradigms of change of criteria, included in the exploration of executive functions $(50,51)$. The instrument used in this paper, the ROCFT, is a complex test, with multiple indexes, whose quantitative and qualitative assessment provides interesting information (38).

From a clinical point of view, the BMI showed an inverse relationship with the copy and memory time of the ROCFT, which supports the difficulty of executive processing in patients with lower BMI. BMI has been suggested as a predictor of treatment outcome in AN $(52,53)$ and is the most common physical criteria used as an indicator $(52,54,55)$. One possible interpretation is that both the BMI and the time of evolution, would be confounding factors, with the status being more relevant, which could be related to certain psychopathological features of eating disorders. In fact, the status was associated with copy time in patients who were studied: patients with evident disorder differed significantly from those in total or partial remission in terms of the time making the copy of the ROCFT. This could suggest that there is a slowdown in the processing of information in patients with worse clinical status, as shown by the decrease in flexibility in tasks when performing the tests $(7,48,56-59)$ with respect to the general population, as well as a greater tendency to pay excessive attention to details and difficulty for global integration (59-61).

Regarding scores on the accuracy of the copy, it was higher than expected with respect to the reference population, which seems to be related to perfectionism. However, the average score in the memory of the copy was lower than the general population, probably due to deficits in nonverbal memory and poverty in visuospatial integration, already described in other ED studies $(8,47,60)$.

Models that integrate clinical psychopathological and variables associated with attitude towards change in eating disorders.

Patients who did not even consider that they had a problem remained in the "Precontemplation" phase and tended to need more time for the performance of complex cognitive processes that require more 
planning and coordination, evaluated by the ROCF. The same happened with the patients who were in "Contemplation" and "Decision", that is, in the less mobilizing stages for change.

On the other hand, although no statistical significance was found in the multivariate model, in the qualitative analysis of the copy, some interesting findings were found in relation to the "Contemplation" and "Action" subscale. Regarding the first, more commonly in adults there seems to be a greater relationship between high scores in types I and II. In addition, patients in the action phase may show greater cognitive flexibility. This could support the idea that in people who manage to consider changes in relation to the disease there seems to be a better cognitive functioning, which could weigh heavily due to the prognostic value of the attitude towards change.

\section{Limitations}

Some limitations deserve special mention, such as the small sample size considering the heterogeneity of the population studied, as well as the existence of $30 \%$ that did not complete all the questionnaires. The absence of statistically significant differences between people who did not complete all the tests and those who did, suggest that the abandonment was not related to neuropsychological variables or the clinical situation.

The relationship between taking psychotropic drugs and attention and cognitive speed, as well as the fact that patients taking psychotropic drugs are generally those with the greatest comorbidity or risk, lead to the consideration that this finding is a possible confounding factor in the study. Other unfavourable effects of depressive symptomatology at the beginning of the therapeutic approach on executive functions have been described in other studies (14).

\section{Conclusions}

Neuropsychological alterations in the population studied and their relationship with some factors which are considered precipitating or maintaining, underline the importance of neuropsychological evaluation in people with eating disorders. These alterations could be summarized as the working memory is lowered and the interference is increased, which is associated with a more controlling and less flexible attitude, and scores in inhibitory care are lower, although, sustained care is preserved in most patients. The Rey Complex Figure Test shows a visoperceptive deficit in some patients.

\section{Clinical impact and future lines}

The global analysis of the sample shows promising results regarding the relationship between dysfunction in some executive functions in ED, especially the central coherence, indicated in previous studies already mentioned. The findings of the present study also support the transdiagnostic perspective, since the DSM-5 diagnosis of the participants was not as relevant as other clinical variables related to the duration of the disorder. 
The findings indicate that those elements related to excessive attention to detail, the loss of a global perception of the situation, or the capacity for attentional maintenance, would correspond to variables related to greater therapeutic resistance, reflecting the low benefit of traditional cognitive behavioural techniques. An evaluation is necessary from a holistic perspective that would allow the incorporation of cognitive rehabilitation into its treatment elements.

\section{List Of Abbreviations}

Attitudes Towards Change in Eating Disorders Scale ACTA

Anorexia nervosa

AN

Beck's depression inventory

$\mathrm{BDI}$

Binge eating disorder

BED

Body Mass Index

BMI

Body Shape Questionnaire

BSQ

Bulimia nervosa

$\mathrm{BN}$

Bulimic Investigatory Test, Edinburgh BITE

Diagnostic and Statistical Manual of Mental Disorders, Fifth Edition DSM-5

Dissociative Experiences Scale DES

Eating disorders

ED

Eating Disorders Inventory EDI

Eating disorder not otherwise specified EDNOS

Letter Number Sequencing

LNS

Rey-Osterrieth complex figure test

ROCFT

State-Trait Anxiety Inventory

STAI

Symbol and Digit Modalities Test

SDMT

Wechsler Adult Intelligence Scale

WAIS

\section{Declarations}


Ethics approval and consent to participate: Informed consent was signed and Local Clinical Research Ethics Committee (Hospital General Universitario from Ciudad Real) approved the study.

Consent for publication: Not applicable.

Availability of data and materials: The datasets used and/or analysed during the current study are available from the corresponding author on reasonable request.

Competing interests: The authors declare that they have no competing interests.

Funding: Not applicable.

Authors' contributions: BMS collected, analysed and interpreted the patient data. LBF and TRC supervised every part of the investigation work and were contributors in writing the manuscript. VMM contributed in writing and translating the manuscript. All authors read and approved the final manuscript.

Acknowledgements: To Investigation Department for their statistical and design support. To FPM, MVL and GBM for their help in patients' selection. And finally, to HP for her assistance in English translation.

\section{References}

1. Volpe U, Tortorella A, Manchia M, Monteleone AM, Albert U, Monteleone P. Eating disorders: What age at onset? Psychiatry Res. 2016;238:225-7.

2. Mustelin L, Silén Y, Raevuori A, Hoek HW, Kaprio J, Keski-Rahkonen A. The DSM-5 diagnostic criteria for anorexia nervosa may change its population prevalence and prognostic value. J Psychiatr Res. 2016;77:85-91.

3. Rojo Moreno L, Plumed Domingo J, Conesa Burguet L, Vaz Leal F, Diaz Marsá M, Rojo-Bofill L, et al. Los trastornos de la conducta alimentaria: Consideraciones sobre nosología, etiopatogenia y tratamiento en el siglo XXI. Rev Psiquiatr Salud Ment. 2012;5(3):197-204.

4. Steiger H, Bruce KR. Phenotypes, endophenotypes, and genotypes in bulimia spectrum eating disorders. Can J Psychiatry. 2007;52(4):220-7.

5. Bulik C, Hebebrand J, Keski-Rahkonen A, Klump K, Reichborn-Kjennerud T, Mazzeo S, et al. Genetic epidemiology, endophenotypes, and eating disorder classification. Int J Eat Disord. 2007;40:S52-60.

6. Holliday J, Tchanturia K, Landau S, Collier D, Treasure J. Is Impaired Set-Shifting an Endophenotype of Anorexia Nervosa? Am J Psychiatry. 2005 Dec 1;162(12):2269-75.

7. Tenconi E, Santonastaso P, Degortes D, Bosello R, Titton F, Mapelli D, et al. Set-shifting abilities, central coherence, and handedness in anorexia nervosa patients, their unaffected siblings and healthy controls: exploring putative endophenotypes. World J Biol Psychiatry. 2010 Sep 21;11(6):813-23.

8. Moynihan J, Rose M, van Velzen J, de Fockert J. Local and global visual processing and eating disorder traits: An event-related potential study. Biol Psychol. 2016 Mar;115:27-34. 
9. Hilbert A, Hoek HW, Schmidt R. Evidence-based clinical guidelines for eating disorders: International comparison. Curr Opin Psychiatry. 2017;30(6):423-37.

10. Byrne SM, Fursland A, Allen KL, Watson $H$. The effectiveness of enhanced cognitive behavioural therapy for eating disorders: An open trial. Behav Res Ther. 2013;51:R2-8.

11. Fairburn CG, Cooper Z, Doll HA, O’Connor ME, Palmer RL, Dalle Grave R. Enhanced cognitive behaviour therapy for adults with anorexia nervosa: A UK-Italy study. Behav Res Ther. 2013;51(1):R2-8.

12. Calugi S, El Ghoch M, Dalle Grave R. Intensive enhanced cognitive behavioural therapy for severe and enduring anorexia nervosa: A longitudinal outcome study. Behav Res Ther. 2017;89:41-8.

13. Calugi S, Franchini C, Pivari S, Conti M, El Ghoch M, Dalle Grave R. Anorexia nervosa and childhood sexual abuse: Treatment outcomes of intensive enhanced cognitive behavioural therapy. Psychiatry Res. 2018;262(May 2017):477-81.

14. Tchanturia K, Davies H, Lopez C, Schmidt U, Treasure J, Wykes T. Neuropsychological task performance before and after cognitive remediation in anorexia nervosa: a pilot case-series. Psychol Med. 2008 Sep 26;38(9):1371-3.

15. Rodriguez Campayo M, Beato Fernández L, Rodriguez Cano T, Martinez-Sánchez F. Adaptación española de la escala de evaluación de la imagen corporal de Gardner en pacientes con trastornos de la conducta alimentaria.;[Spanish version of the Gardner body image validation scale in patients with eating behavior disorders]. Actas Esp Psiquiatr. 2003;31(2):59-64.

16. Herzog DB, Sacks NR, Keller MB, Lavori PW, von Ranson KB, Gray HM. Patterns and predictors of recovery in anorexia nervosa and bulimia nervosa. J Am Acad Child Adolesc Psychiatry. 1993 Jul $1 ; 32(4): 835-42$.

17. Raich R, Mora M, Soler A, Ávila C, Clos I, Zapater I. Adaptación de un instrumento de evaluación de la insatisfacción corporal. Clínica y Salud. 1996;7(1):51-6.

18. Corral S, González M, Pereña J, Seisdedos N. EDI-2 - Inventario de trastornos de la conducta alimentaria. Ediciones T, editor. Madrid; 1998.

19. Garner D. Manual Inventario de trastornos de la conducta alimentaria. TEA, editor. Madrid; 1998.

20. Henderson M, Freeman CPL. A self-rating scale for bulimia: The 'BITE’. Br J Psychiatry. 1987;18-25.

21. Rivas T, Bersabe R, Freeman C. Fiabilidad y validez del test de investigación bulímica de Edimburgo (BITE) en muestra de adolescentes españoles. Psicol Conductual. 2004;12(3):447-61.

22. Sanz J, Perdigón AL, Vázquez C. Adaptación española del Inventario para la Depresión de Beck-II (BDI-II): 2.Propiedades psicométricas en población general. Clin y Salud. 2003;14(3):249-80.

23. Consejo general de colegios oficiales de psicólogos. Evaluación del inventario BDI-II.

24. Spielberger CD, Gonzalez-Reigosa F, Martinez-Urrutia A, Natalicio LFS, Natalicio DS. Development of the Spanish edition of the State-Trait Anxiety Inventory. Interam J Psychol. 1971;5:145-158.

25. Spielberger C, Gorsuch R, Lushene R. State-Trait Anxiety Inventory (self evaluation Questionnaire). Palo Alto, California: Consulting Psychologists Press; 1970. 
26. Buela-Casal G, Guillén A, Guillén-Riquelme A, Seisdedos- Cubero N. Manual Cuestionario de Ansiedad Estado-Rasgo Adaptación española. Madrid; 2015.

27. Icaran E, Colom R, Orengo Garcia F. Dissociative experiences: A measurement scale. Exp disociativas una escala medida. 1996;70(70):69-84.

28. Bernstein EM, Putnam FW. Development, reliability, and validity of a dissociation scale. Vol. 174, The Journal of Nervous \& Mental Disease. 1986. p. 727-35.

29. Carlson EB, Putnam FW. An Update on the Dissociative Experiences Scale. Dissociation. 1993;6(1):16-27.

30. Bernstein Carlson E, Putnam FW, Ross CA, Torem M. Validity of the Dissociative Experiences Scale in screening for multiple personality disorder: A multicenter study. Am J Psychiatry. 1993;150(7):10306.

31. Tobergte DR, Curtis S. Escala de experiencias disociativas. J Chem Inf Model. 2013;53(9):1689-99.

32. Beato Fernández L, Rodríguez Cano T. El cuestionario de actitudes frente al cambio en los trastornos de la conducta alimentaria (ACTA). Desarrollo y propiedades psicométricas. Actas Españolas Psiquiatr. 2003;31(3):111-9.

33. Campos Amador JA. Escala de inteligencia de Wechsler para adultos-IV (WAIS-IV). 2013.

34. Stroop J. Studies of interference in serial verbal reactions. J Exp Psychol. 1935;18:643-62.

35. Golden CJ. Stroop. Test de colores y palabras. 5a . Ediciones TEA, editor. Madrid; 1993.

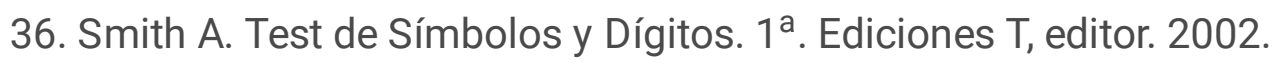

37. Smith A. Symbol Digit Modalities Test (SDMT). Manual (Revised). Services WP, editor. Los Angeles; 1982.

38. Rey A. Test de copia y reproducción de memoria de figuras geométricas complejas. 9a . Ediciones TEA, editor. Madrid; 2009.

39. Rey A. Test de copie et reproduction de mémoire de figures géométriques complexes. Appliquée LE du C de P, editor. Paris; 1959.

40. Lang K, Roberts M, Harrison A, Lopez C, Goddard E, Khondoker M, et al. Central coherence in eating disorders: A synthesis of studies using the rey osterrieth complex figure test. PLoS One. 2016;11(11):1-11.

41. Lopez C, Tchanturia K, Stahl D, Booth R, Holliday J, Treasure J. An examination of the concept of central coherence in women with anorexia nervosa. Int J Eat Disord. 2008;41(2):143-52.

42. Booth R. Local-global processing and cognitive style in autism spectrum disorders and typical development. University of London; 2006.

43. Oltra-Cucarella J, Espert R, Rojo L, Jacas C, Guillén V, Moreno S. Neuropsychological impairments in anorexia nervosa: A spanish sample pilot study. Appl Neuropsychol Adult. 2013;0:1-15.

44. Frank GKW. Advances from neuroimaging studies in eating disorders. CNS Spectr. 2015;20(4):391400. 
45. Donnelly B, Touyz S, Hay P, Burton A, Russell J, Caterson I. Neuroimaging in bulimia nervosa and binge eating disorder: A systematic review. J Eat Disord. 2018;6(1).

46. Smith KE, Mason TB, Johnson JS, Lavender JM, Wonderlich SA. A systematic review of reviews of neurocognitive functioning in eating disorders: The state-of-the-literature and future directions. Vol. 51, International Journal of Eating Disorders. John Wiley and Sons Inc.; 2018. p. 798-821.

47. Van den Eynde F, Guillaume S, Broadbent H, StahI D, Campbell IC, Schmidt U, et al. Neurocognition in bulimic eating disorders: A systematic review. Acta Psychiatr Scand. 2011 Aug;124(2):120-40.

48. Weider S, Indredavik MS, Lydersen S, Hestad K. Neuropsychological function in patients with anorexia nervosa or bulimia nervosa. Int J Eat Disord. 2015 May;48(4):397-405.

49. Tchanturia K, Lock J. Cognitive Remediation Therapy for Eating Disorders: Development, Refinement and Future Directions. Curr Top Behav Neurosci. 2011;6:269-87.

50. Tchanturia K, Davies H, Roberts M, Harrison A, Nakazato M, Schmidt U, et al. Poor cognitive flexibility in eating disorders: examining the evidence using the Wisconsin Card Sorting Task. García AV, editor. PLoS One. 2012 Jan 12;7(1):e28331.

51. Lock J, Garrett A, Beenhakker J, Reiss AL. Aberrant Brain Activation During a Response Inhibition Task in Adolescent Eating Disorder Subtypes. Am J Psychiatry. 2011 Jan 1;168(1):55-64.

52. Herzog DB, Dorer DJ, Keel PK, Selwyn SE, Ekeblad ER, Flores AT, et al. Recovery and Relapse in Anorexia and Bulimia Nervosa: A 7.5-Year Follow-up Study. J Am Acad Child Adolesc Psychiatry. 1999 Jul;38(7):829-37.

53. Franko DL, Tabri N, Keshaviah A, Murray HB, Herzog DB, Thomas JJ, et al. Predictors of long-term recovery in anorexia nervosa and bulimia nervosa: Data from a 22-year longitudinal study. $J$ Psychiatr Res. 2018;96(July 2017):183-8.

54. Bardone-Cone AM, Hunt RA, Watson HJ. An Overview of Conceptualizations of Eating Disorder Recovery, Recent Findings, and Future Directions. Curr Psychiatry Rep. 2018 Sep 9;20:79.

55. Asociación Americana de Psiquiatría. Manual diagnóstico y estadístico de los trastornos mentales (DSM-5). 5a . Arlington V, editor. 2014.

56. Hatch A, Madden S, Kohn MR, Clarke S, Touyz S, Gordon E, et al. Emotion brain alterations in anorexia nervosa: A candidate biological marker and implications for treatment. 2010 Jul;35(4):26774.

57. Galimberti E, Fadda E, Cavallini MC, Martoni RM, Erzegovesi S, Bellodi L. Executive functioning in anorexia nervosa patients and their unaffected relatives. Psychiatry Res. 2013 Aug 15;208(3):23844.

58. Phillipou A. Comprehensive neurocognitive assessment of patients with anorexia nervosa. World J Psychiatry. 2015;5(4):404.

59. Allen KL, Byrne SM, Hii H, Eekelen A V, Mattes E, Foster JK. Neurocognitive functioning in adolescents with eating disorders: A population-based study. Cogn Neuropsychiatry. 2013;18(5):355-75. 
60. Darcy AM, Fitzpatrick KK, Manasse SM, Datta N, Klabunde M, Colborn D, et al. Central coherence in adolescents with bulimia nervosa spectrum eating disorders. Int J Eat Disord. 2015 Jul;48(5):48793.

61. López B, Leekam SR. Teoría de la coherencia central: una revisión de los supuestos teóricos. Infanc y Aprendiz. 2007 Jan 23;30(3):439-57. 